\title{
miR-874 regulates multiple-drug resistance in gastric cancer by targeting ATG16L1
}

\author{
HAIJIN HUANG $^{1 *}$, JIE TANG $^{2 *}$, LEI ZHANG ${ }^{2 *}$, YANZHI BU ${ }^{3}$ and XIAOYU ZHANG ${ }^{4}$ \\ ${ }^{1}$ Department of General Surgery, Hongze District People's Hospital, Huai'an, Jiangsu 223100; \\ ${ }^{2}$ Department of Pediatric Surgery, Children's Hospital of Nanjing Medical University, Nanjing, Jiangsu 210000; \\ ${ }^{3}$ Department of General Surgery, Lianshui Country People's Hospital, Lianshui Country, Huai'an, Jiangsu 223400; \\ ${ }^{4}$ Department of General Surgery, Huai'an Second People's Hospital and \\ The Affiliated Huai'an Hospital of Xuzhou Medical University, Huai'an, Jiangsu 223001, P.R. China
}

Received May 9, 2018; Accepted August 13, 2018

DOI: $10.3892 /$ ijo.2018.4593

\begin{abstract}
Chemotherapy is an important treatment option for gastric cancer (GC); however, chemotherapy usually fails due to drug resistance, particularly multidrug resistance (MDR). In our previous studies, microRNA (miR)-874 was demonstrated to serve an important role in tumour growth, apoptosis and angiogenesis. In the present study, the precise roles and underlying mechanisms of miR-874 in MDR were investigated in GC. The overexpression of miR-874 reversed cancer cell drug resistance in vitro. According to reporter gene and western blot assays, Autophagy-related 16-like 1 (ATG16 L1) was identified as a direct target of miR-874. ATG16L1 was also demonstrated to be positively associated with autophagy. Reducing the expression of ATG16L1 and inhibiting the occurrence of autophagy sensitized GC cells to chemotherapy. Thus, the miR-874/ATG16L1/autophagy regulatory loop was demonstrated to serve an important role in MDR in GC. Furthermore, miR-874 may be used as a prognostic factor in GC. Overall, miR-874 could inhibit autophagy and sensitize GC cells to chemotherapy via the target gene ATG16L1, highlighting the potential clinical application of miR-874 in chemotherapeutic resistance.
\end{abstract}

Correspondence to: Dr Yanzhi Bu, Department of General Surgery, Lianshui Country People's Hospital, 6 Hongri Road, Lianshui Country, Huai'an, Jiangsu 223400, P.R. China

E-mail: 1291221279@qq.com

Dr Xiaoyu Zhang, Department of General Surgery, Huai'an Second People's Hospital and The Affiliated Huai'an Hospital of Xuzhou Medical University, 62 Huaihai South Road, Huai'an, Jiangsu 223001, P.R. China

E-mail: yllzxy@163.com

${ }^{*}$ Contributed equally

Key words: miR-874, multiple-drug resistance, gastric cancer, autophagy-related 16-like 1

\section{Introduction}

During the past century, gastric cancer (GC) has remained the fourth most prevalent type of malignant cancer and the second leading cause of cancer-associated mortality worldwide (1). Currently, the majority of patients with GC are diagnosed at the advanced stage of the disease due to the lack of effective diagnostic methods at the early stages. The five-year survival rate of advanced patients with GC is only $5-20 \%$, and the median overall survival is $<1$ year (2). For advanced-stage patients, chemotherapy is the first-line treatment. Unfortunately, chemotherapy resistance is common, particularly multidrug resistance (MDR), which affects treatment and prognosis $(3,4)$. Although the mechanisms underlying MDR have been extensively explored, the key multiple-drug resistance features of this clinical phenomenon remain unclear.

Micro (mi)RNAs are short (20-24 nt), stable, non-coding RNA molecules that negatively regulate $60 \%$ of coding genes by targeting the 3' untranslated regions (3'UTRs) of specific mRNAs to prevent translation and/or promote degradation (5). Accumulating evidence indicates that miRNAs serve important roles in chemoresistance in various types of cancer, including GC (6-14). However, the exact mechanisms underlying the regulation of chemoresistance in GC by miRNAs remain unclear. miR- 874 has been reported in numerous types of cancer, including $\mathrm{GC}$, hepatocellular carcinoma, colorectal cancer, breast cancer, non-small-cell lung cancer, and head and neck squamous carcinoma $(8,15-18)$. In our previous studies, miR-874 was demonstrated to serve a potential role in tumour growth, apoptosis and angiogenesis in GC. However, to the best of our knowledge, the association between miR-874 and chemotherapy drug resistance in GC has not been reported. The present study demonstrated that miR-874 enhanced the sensitivity of GC cells to chemotherapy. Furthermore, autophagy-related 16-like 1 (ATG16L1) was identified as a direct and functional target of miR-874. In addition, ATG16L1 expression was revealed to be increased in GC MDR cells, and positively associated with autophagy and chemotherapy resistance. Lastly, it was demonstrated that miR-874 expression was downregulated in chemoresistant 
patients, and was associated with ATG16L1 expression and overall survival in patients with GC.

\section{Materials and methods}

Tissue samples. The acquisition of tissue specimens and the study protocol were performed in strict accordance with the regulations of the Institutional Review Board of Xuzhou Medical University (Xuzhou, China). All patients signed informed consent forms. Human GC specimens were collected between January 2010 and December 2012, and detailed clinicopathological and follow-up information were obtained from the Tissue Sample Centre of the Affiliated Huai'an Hospital at Xuzhou Medical University in China. In all cases, the diagnoses and grading were confirmed by two experienced pathologists and were performed according to the criteria of the American Joint Committee on Cancer $(19,20)$.

Cells and cell culture. The human GC cell lines SGC7901, BGC823 (Type Culture Collection of the Chinese Academy of Sciences, Shanghai, China), AGS (American Type Culture Collection, Manassas, VA, USA), and SGC7901/cisplatin (DDP) (established and maintained in our laboratory) were cultured in RPMI-1640 supplemented with $10 \%$ foetal bovine serum (both from Wisent Biotechnology, Nanjing, China) and antibiotics (1\% penicillin/streptomycin) (Gibco; Thermo Fisher Scientific, Inc., Waltham, MA, USA). All cell lines were cultured in a humidified chamber supplemented with $5 \% \mathrm{CO}_{2}$ at $37^{\circ} \mathrm{C}$. Cell resistance was induced by gradually increasing the cisplatin concentration in the culture medium. SGC-7901 cells in the logarithmic growth phase were seeded in the culture medium, which contained DDP (Sigma-Aldrich; Merck KGaA, Darmstadt, Germany) at a low starting concentration of $0.05 \mu \mathrm{g} / \mathrm{ml}$. After $48 \mathrm{~h}$, the culture medium was discarded, fresh medium was added and culturing was continued. Once normal growth was observed, the cells were continuously treated with $0.05 \mu \mathrm{g} / \mathrm{ml}$ DDP for $48 \mathrm{~h}$ after digestion and passage. The cells were cultured and passaged in this manner, and the DDP concentration was gradually increased (the concentration of DDP was increased by $0.05 \mu \mathrm{g} / \mathrm{ml}$ each time; range, $0.05-1 \mu \mathrm{g} / \mathrm{ml}$ ) to continuously induce the cells. Finally, a cell line that was tolerant of $1 \mu \mathrm{g} / \mathrm{ml}$ DDP was established.

RNA extraction, cDNA synthesis and reverse transcriptionquantitative polymerase chain reaction ( $R T-q P C R)$. Total RNA was extracted from frozen tissues or cells using TRIzol (Invitrogen; Thermo Fisher Scientific, Inc.) according to the manufacturer's protocol. RNA purity was assessed using a Thermo NanoDrop 2000 (NanoDrop Technologies; Thermo Fisher Scientific, Inc., Wilmington, DE, USA) using the standard absorbance ratios of A260/A280 $\geq 1.8$ and A260/A230 $\geq 1.5$. Complementary DNAs were synthesized from $1 \mu \mathrm{g}$ of total RNA using the TaqMan Reverse Transcription kit (Life Technologies; Thermo Fisher Scientific, Inc.) as follows: $42^{\circ} \mathrm{C}$ for $15 \mathrm{~min} ; 85^{\circ} \mathrm{C}$ for $5 \mathrm{sec}$; followed by storage at $4^{\circ} \mathrm{C}$. To determine the mRNA expression of ATG16L1 and miR-874, RT-qPCR was performed on a 7500 Real-Time PCR system (Life Technologies; Thermo Fisher Scientific, Inc.) using Fast Start Universal SYBR-Green Master mix (Roche, Basel, Switzerland) according to the manufacturer's protocol. The following thermocycling conditions were maintained: $95^{\circ} \mathrm{C}$ for $10 \mathrm{~min}$; followed by 40 cycles of $95^{\circ} \mathrm{C}$ for $15 \mathrm{sec}$ and $60^{\circ} \mathrm{C}$ for $1 \mathrm{~min}$. All mRNA and miRNA quantification data were normalized to GAPDH and U6, respectively. All experiments were performed independently in triplicate. The relative expression levels of target genes were normalized to those of the internal control genes using the $2^{-\Delta \Delta \mathrm{Cq}}$ cycle quantification method (21). The primers were as follows: GAPDH forward, 5'-ATCTCTGCCCCCTCTGCTGA-3' and reverse, 5'-GATGACCTTGCCCACAGCCT-3'; miR-874 forward, 5'-GGCCCTGAGGAAGAACTGAG-3' and reverse, 5'-TGAGAT CCAACAGGCCTTGAC-3'; U6 forward, 5'-GCTTCGGCAGCA CATATACTAAAAT-3' and reverse, 5'-CGCTTCACGAATTTG CGTGTCAT-3'; and ATG16L1 forward, 5'-AGGACAGGGAGAT GCAGATGA-3' and reverse, 5'-GATTGGCTTCCTGGGCTTT-3'.

Vector constructs, lentivirus production and cell transfection. The lentiviral vectors has-miR-874-pre-microRNA (pre-miR-874) and has-miR-874-pre-inhibitor (miR-874 inhibitor) were purchased from Shanghai GenePharma Co., Ltd. A scrambled lentiviral construct (miR-NC) was used as a negative control (NC). The sequence of pre-miR-874 was 5'-TTAGCCCTGCGG CCCCACGCACCAGGGTAAGAGAGACTCTCGCTTCCTG CCCTGGCCCGAGGGACCGACTGGCTGGGC-3'. The sequence of miR-874-inhibitor was 5'-GCCCAGCCAGTCG GTCCCTCGGGCCAGGGCAGGAAGCGAGAGTCTCTCT TACCCTGGTGCGTGGGGCCGCAGGGCTAA-3'. The sequence of miR-NC was 5'-TTGTATACAAAAGTACTGGC ATGAAAACACATCATGTTATGTACTACACATTATACTG GTCAGAACACATATGTTTAA-3' The lentiviral vectors [multiplicity of infection (MOI) of 15] were used to transfect GC cells once they had grown to $40-50 \%$ confluence with polybrene (hexadimethrine bromide) $(5 \mu \mathrm{g} / \mathrm{ml})$ (Sigma-Aldrich; Merck $\mathrm{KGaA}$ ). After culturing for $8 \mathrm{~h}$, the culture medium was replaced with fresh medium. Puromycin culturing was used to select the aforementioned stable cell lines. Subsequently, the expression of miR-874 was detected to confirm the efficiency of transfection.

A lentiviral vector containing an shRNA targeting human ATG16L1 was also purchased from Shanghai GenePharma Co., Ltd. The target sequence of ATG16L1-shRNA was 5'-GAT TACTGCCCTGGACTTAAA-3'. The sequence of 5'-CAGTAC TTTTGTGTAGTACAA-3' was used as a negative control (sh-NC). The cells were infected and selected as aforementioned. RT-qPCR and western blotting was then performed to detect the expression of ATG16L1, and confirm the efficiency of transfection.

In vitro and in vivo drug sensitivity assays. Each well of a 96-well plate was seeded with $5 \times 10^{3}$ cells (SGC7901, SGC7901-NC, SGC7901-inhibitor, SGC7901/DDP, SGC7901/DDP-NC,SGC7901/DDP-pre,SGC7901/DDP-sh-NC, SGC7901/DDP-sh-ATG16L1 or SGC7901/DDP+CQ). After $24 \mathrm{~h}$, culture media containing different concentrations of the chemotherapeutic drugs DDP $(0-25 \mu \mathrm{g} / \mathrm{ml}), 5$-fluorouracil (5-FU) $(0-25 \mu \mathrm{g} / \mathrm{ml})$ and vincristine (VCR) $(0-50 \mu \mathrm{g} / \mathrm{ml})$ (both from Sigma-Aldrich; Merck KGaA) were added to each well. After the plates were incubated for 48 h, a Cell Counting Kit 8 (CCK8; Dojindo Molecular Technologies, Inc., Kumamoto, Japan) assay was performed. The half maximal inhibitory concentration $\left(\mathrm{IC}_{50}\right)$ of each drug was calculated. For the in vivo experiments, $\sim 2.0 \times 10^{6}$ cells stably transfected with pre-miR-874 
or NC were inoculated subcutaneously into both flanks of nude mice. After 2 weeks, the mice were intraperitoneally injected with PBS containing 5-FU or DDP $(10 \mathrm{mg} / \mathrm{kg}$ ) once weekly. The tumour size on their skin surfaces was measured once weekly. The mice were humanely euthanized on day 28 , and the tumours were measured and images. A total of six female nude mice (BALB/c nude mice, 5-weeks old, 15-18 g) were purchased from Shanghai Experimental Animal Centre (Shanghai, China) and housed under specific pathogen-free conditions. All animal experiments were conducted according to the recommendations of the Xuzhou Medical University Institutional Animal Care and Use Committee (approval no. IACUC-201601012).

3'UTR luciferase constructs and assay. The 3'UTR of ATG16L1 mRNA containing either the putative or mutated miR-874 binding site was synthesized by Sangon Biotech Co., Ltd. (Shanghai, China). The cells were co-transfected with plasmids (Shanghai GenePharma Co., Ltd.) expressing wild-type ATG16L1 or mutant ATG16L1 in addition to cells stably transfected with pre-miR-874 or miR-NC. After $36 \mathrm{~h}$ of transfection, firefly and Renilla luciferase activities were measured using the Dual-Luciferase Reporter assay kit (Promega Corporation, Madison, WI, USA).

Transmission electron microscopy (TEM). Cells were collected and centrifuged at $150 \mathrm{xg}, 4^{\circ} \mathrm{C}$ for $10 \mathrm{~min}$ and then fixed in $2.5 \%$ glutaraldehyde in $0.1 \mathrm{M}$ phosphate buffer ( $\mathrm{pH} 7.2)$ overnight at $4^{\circ} \mathrm{C}$. Next, the cells were fixed in $1 \% \mathrm{OsO}_{4}$ for $1 \mathrm{~h}$ at room temperature. Subsequently, the samples were dehydrated using an increasing concentration gradient of ethanol (50-100\%) and the cells were then embedded in Epon. The samples were cut into ultrathin $(50 \mathrm{~nm})$ sections and counterstained with $0.3 \%$ lead citrate at room temperature for $20 \mathrm{~min}$. Images were generated using a JEM-1010 electron microscope at a magnification of $\mathrm{x} 10,000$ and $\mathrm{x} 40,000$.

Western blotting. Protein extract was obtained using RIPA Lysis buffer (Beyotime Institute of Biotechnology, Haimen, China) containing $1 \%$ phenylmethylsulfonyl fluoride (Sigma-Aldrich; Merck KGaA). The protein concentration of cell lysate was determined using an Enhanced BCA Protein assay kit (Beyotime Institute of Biotechnology). Equal amounts (30 $\mu \mathrm{g}$ protein/lane) of samples were size-fractioned on a $10 \%$ SDS-PAGE gel and transferred to polyvinylidene difluoride membranes (Bio-Rad Laboratories, Inc., Hercules, CA, USA). The membranes were blocked with $5 \%$ non-fat milk in Tris-buffered saline for $2 \mathrm{~h}$ at room temperature, and then incubated with specific antibodies at $4^{\circ} \mathrm{C}$ overnight. Following washing with TBS-Tween-20 for $15 \mathrm{~min}$ at room temperature, the membranes were incubated with horseradish peroxidase (HRP)-conjugated secondary antibodies for $2 \mathrm{~h}$ at room temperature. The proteins were visualized using a SuperSignal West Femto Maximum Sensitivity Substrate kit (Thermo Fisher Scientific, Inc.). The software Image-Pro Plus (version 6.0; Media Cybernetics, Inc., Rockville, MD, USA) was used to quantify protein expression. Mouse anti-human GAPDH primary antibodies (cat. no. SC-365062; dilution 1:1,000) were purchased from Santa Cruz Biotechnology, Inc. (Dallas, TX, USA). Goat anti-human ATG16L1 primary antibodies (cat. no. ab223238; dilution 1:1,000) were purchased from Abcam (Cambridge, UK) and rabbit anti-human LC3I/II primary antibodies (cat. no. 4108; dilution 1:1,000) were purchased from Cell Signaling Technology, Inc. (Danvers, MA, USA). The HRP-conjugated second antibodies used were as follows: Donkey anti-goat IgG (cat. no. A0181; dilution 1:1,000), goat anti-rabbit IgG (cat. no. A0208; dilution 1:1,000) and goat anti-mouse IgG (cat. no. A0216; dilution 1:1,000) (all from Beyotime Institute of Biotechnology).

Immunohistochemistry (IHC). All specimens were fixed in $4 \%$ formalin at temperature for $24 \mathrm{~h}$ and embedded in paraffin prior to performing the IHC analysis, as described in detail in our previous report (18). The specimens were examined in a blinded manner. Five fields were selected for examination, and the percentage of positive tumour cells and the cell-staining intensity were determined at the magnification of $\mathrm{x} 200$ and x400 with a Nikon Eclipse 90i digital microscope (Nikon Corporation, Tokyo, Japan).

Bioinformatics. TargetScan (http://www.targetscan.org), miRanda (http://www.microrna.org/microrna/home.do), miRBase (http://www.mirbase.org), miRDB (http://www. mirdb.org) and CLIPdb (http://clipdb.ncrnalab.org) were used to predict the genes targeted by miR- 874 .

Chloroquine $(C Q)$. The autophagy inhibitor CQ (Sigma-Aldrich; Merck KGaA) was used to examine whether autophagy affected chemoresistance in GC cells. Cells were treated with or without CQ $(20 \mu \mathrm{mol} / \mathrm{l})$ for $24 \mathrm{~h}$ prior to the other experiments.

Statistical analysis. SPSS software (version 18.0; SPSS, Inc., Chicago, IL, USA) was used to perform statistical analyses. Student's t-test (two-tailed) or one-way analysis of variance followed by the Student-Newman-Keuls post-hoc test was performed to analyse the in vitro and in vivo data. The clinicopathological factors were compared by performing unpaired t-tests or Pearson's $\chi^{2}$ tests. The quantitative data are presented as the mean \pm standard deviation. The Kaplan-Meier method was used to estimate the survival curve. The differences in the survival distributions were determined by performing log-rank tests. $\mathrm{P}<0.05$ was considered to indicate a statistically significant difference.

\section{Results}

miR-874 regulates the sensitivity of GC cells tochemotherapeutic drugs in vitro. First, RT-qPCR analysis was performed to detect miR-874 expression in SGC7901 and SGC7901/DDP cells. miR-874 expression was significantly downregulated in SGC7901/DDP cells compared with SGC7901 cells (Fig. 1A). To investigate the effect of miR-874 on chemotherapeutic resistance, miR-874 overexpression and knockdown cell lines were established. SGC7901 and SGC7901/DDP cells were transfected with miR-874 inhibitors or pre-miR-874. miR-874 inhibitor transfection resulted in miR-874 being knocked down by $\sim 80 \%$ in SGC7901 cells compared with the NC control group (Fig. 1B). Furthermore, miR-874 was significantly increased by $\sim 30$-fold in SGC7901/DDP cells following transfection with pre-miR-974 compared with the NC control (Fig. 1C). CCK8 assays were then performed to generate 

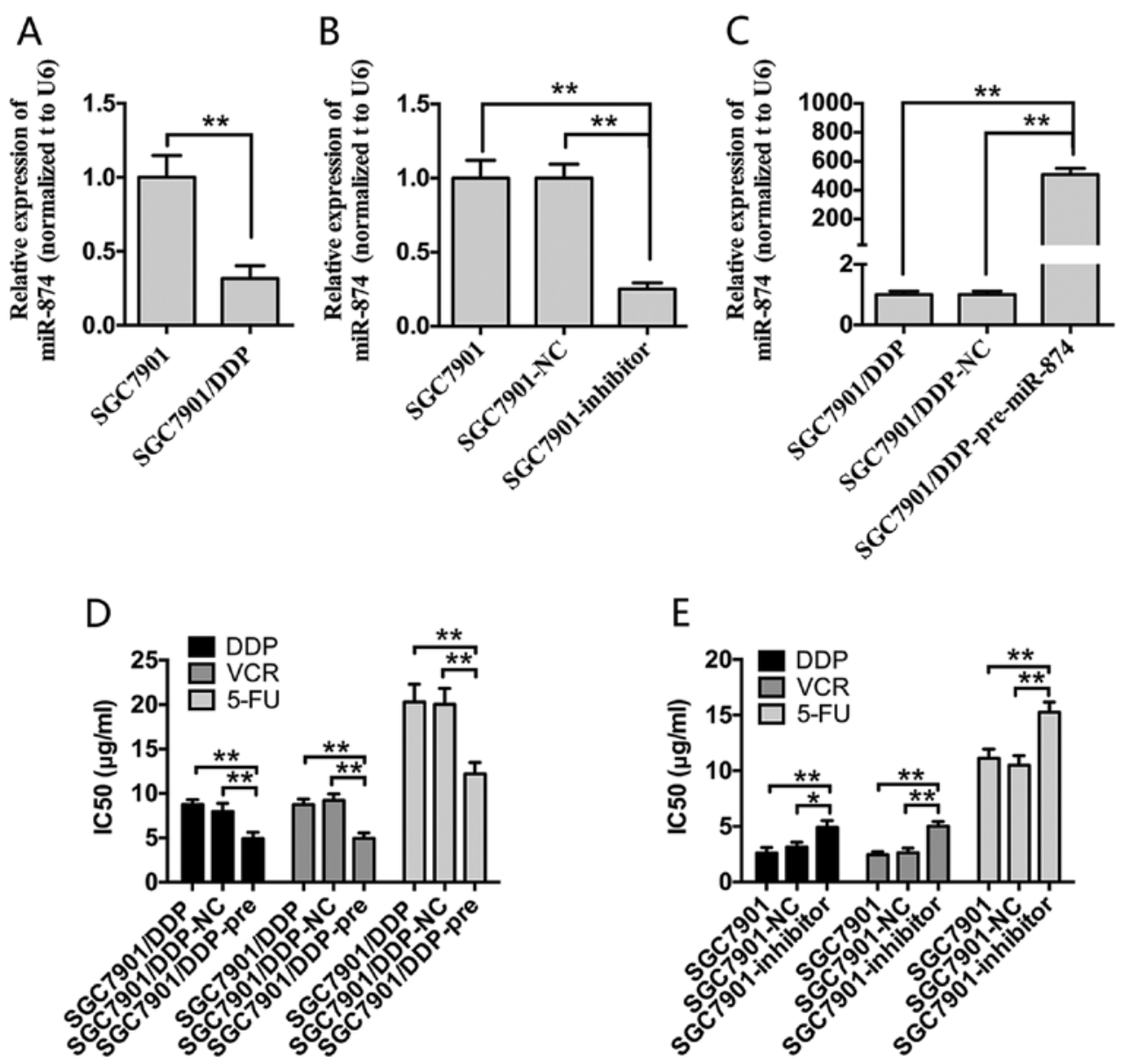

Figure 1. Expression and function of miR-874 in gastric cancer cells. (A) The downregulation of miR-874 was confirmed by reverse transcription-quantitative polymerase chain reaction in SGC7901/DDP cells. (B) SGC7901 cells were transfected with miR-874 inhibitors or NC, and then the expression of miR-874 was detected. (C) The expression of miR-874 was detected after the pre-miR-874 or NC were transfected into SGC7901/DDP cells. (D) The IC 50 values were detected after pre-miR-874 or NC were transfected into SGC7901/DDP cells to evaluate the capacity of miR-874 in regulating the chemotherapy sensitivity. (E) The $\mathrm{IC}_{50}$ values were detected after miR-874-inhibitors or NC were transfected into SGC7901 cells. ${ }^{* *} \mathrm{P}<0.01$. DDP, cisplatin; NC, negative control; miR, microRNA; $\mathrm{IC}_{50}$, half maximal inhibitory concentration; VCR, vincristine; 5-FU, 5-fluorouracil.

cell growth curves and calculate the $\mathrm{IC}_{50}$ values. Overexpression of miR-874 significantly enhanced the sensitivity of SGC7901/DDP cells to DDP, VCR and 5-FU compared with the blank control and NC groups. Conversely, transfection of SGC7901 cells with inhibitors of miR-874 significantly increased the $\mathrm{IC}_{50}$ values of these three chemotherapeutic agents compared with the two control groups (Fig. 1D and E).

ATG16L1 is a direct target of miR-874. ATG16L1 was identified as a potential target gene of miR-874 through bioinformatic algorithms. RT-qPCR and western blot assays were performed, which revealed that ATG16L1 was significantly upregulated in SGC7901/DDP cells compared with SGC7901 cells (Fig. 2A-C). Furthermore, the overexpression of miR-874 reduced the expression of ATG16L1 at the mRNA and protein levels in SGC7901/DDP cells (Fig. 2D and E), whereas the downregulation of miR-874 led to the opposite effect (Fig. 2E and F). A putative miR-874 binding site was identified within the 3'UTR of ATG16L1 (Fig. 2G). A dual-luciferase reporter system was used to validate whether ATG16L1 is a direct target of miR-874. Wild-type (Wt) and mutant $(\mathrm{Mu})$ versions of the ATG16L1 3'UTR were cloned into the reporter plasmids. Forced expression of miR- 874 significantly suppressed luciferase activity from the wild-type reporter, but did not affect the mutant reporter (Fig. 2H). Thus, ATG16L1 is likely a direct target of miR-874. To further explore the function of ATG16L1 in MDR in GC, a shRNA targeting ATG16L1 was constructed and introduced using lentiviral gene transfer, and its inhibitory effect was confirmed by RT-qPCR and western blot assays (Fig. 3A-C). Remarkably, the silencing of ATG16L1 sensitized SGC7901/DDP cells to chemotherapeutic agents and significantly decreased the $\mathrm{IC}_{50}$ values of these drugs (Fig. 3D). Thus, ATG16L1 likely serves an important role in GC as a direct functional target gene of miR-874.

Chemoresistant GC cells exhibit increased autophagy. ATG16L1 is a member of a large protein complex that is necessary for autophagy (22). As the expression of ATG16L1 was demonstrated to be increased at the mRNA and protein levels, we hypothesized that autophagy may be enhanced in SGC7901/DDP cells. To test this hypothesis, TEM was performed to evaluate autophagosomes. Compared with SGC7901 cells, autophagosomes markedly accumulated in the cytoplasm of SGC7901/DDP cells (Fig. 4A). The processing of the LC3-I protein to LC3-II, which is a hallmark of autophagy, was also evaluated by performing western blot analysis. A 
A

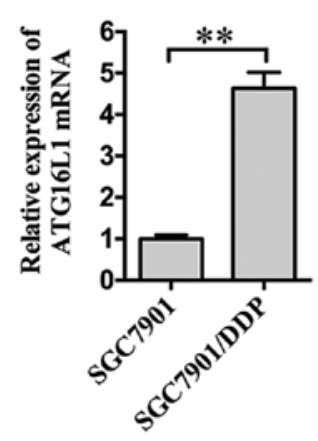

D

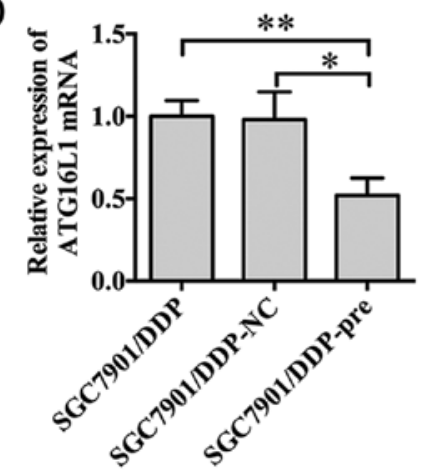

E

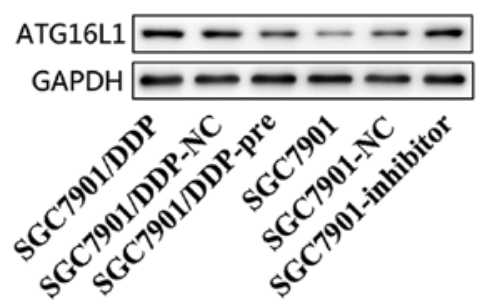

G

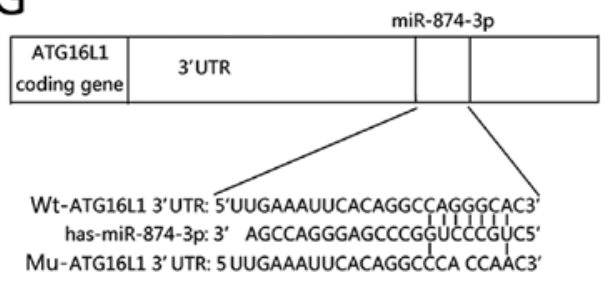

B

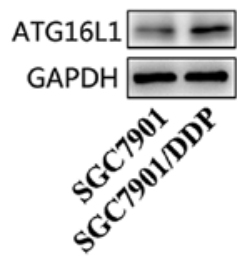

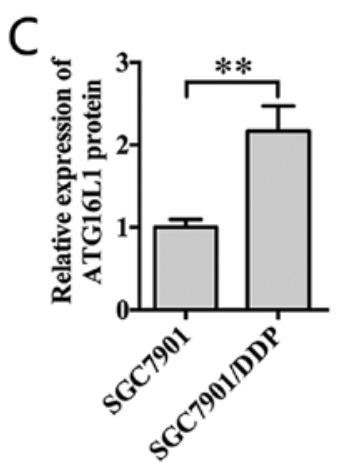

F

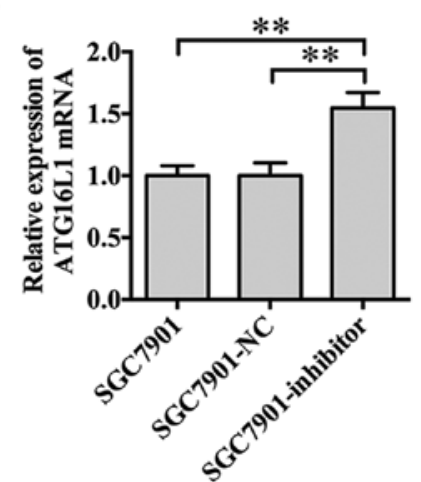

$\mathrm{H}$

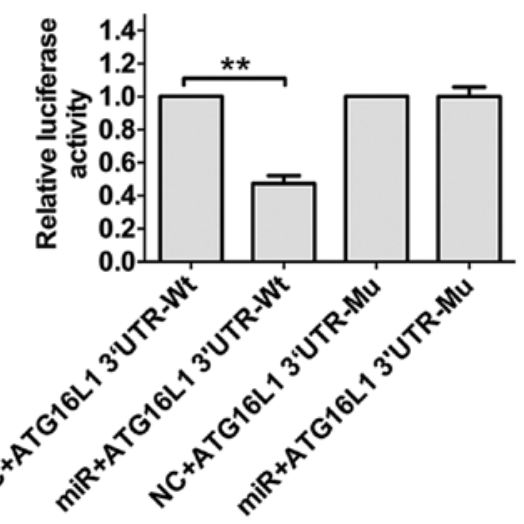

Figure 2. ATG16L1 is direct target gene of miR-874. (A) The expression of ATG16L1 mRNA was detected by RT-qPCR analyses in SGC 7901 and SGC7901/DDP cells. (B) The expression of ATG16L1 protein was detected through western blot assays in SGC7901 and SGC7901/DDP cells. (C) The histogram shows the relative protein expression of ATG16L1. (D) SGC7901/DDP cells were transfected with pre-miR-874 or NC, and the expression of ATG16L1 mRNA was then evaluated by RT-qPCR. (E) The protein expression of ATG16L1 was detected in different group of cells (SGC7901/DDP; SGC7901/DDP-NC; SGC7901/DDP-pre; SGC7901; SGC7901-NC; SGC7901-inhibitor). (F) SGC7901 cells were transfected with miR-874 inhibitors or NC, and the expression of ATG16L1 mRNA was then evaluated by RT-qPCR. (G) The sequences of Wt-ATG16L1 or Mu-ATG16L1 3'UTR and the binding sites was shown. (H) Dual luciferase assays were conducted in SGC7901/DDP cells after co-transfection with wild-type or mutant ATG16L1 3'UTR plasmids and NC or pre-miR-874. ${ }^{*} \mathrm{P}<0.05$ and ${ }^{* *} \mathrm{P}<0.01$. DDP, cisplatin; NC, negative control; miR, microRNA; Mu, mutant; Wt, wild type; UTR, untranslated region; ATG16L1, autophagy-re lated 16-like 1.

marked increased expression of LC3-II in SGC7901/DDP cells was observed compared with that in SGC7901 cells (Fig. 4B). The results suggested that the chemoresistant GC cells exhibited increased autophagy.

ATG16L1 inhibits chemosensitivity in chemoresistant GC cells by promoting autophagy. The silencing of ATG16L1 increased the sensitivity of SGC7901/DDP cells to chemotherapeutic agents. Next, whether ATG16L1 regulated autophagy was investigated. According to western blot analysis, autophagy was markedly decreased in SGC7901/DDP cells in which ATG16L1 was silenced compared with the NC-transfected group (Fig. 4C). The expression of LC3-II was markedly increased in SGC7901/DDP cells following CQ treatment for 24 h compared with the untreated control group (Fig. 4D). Additionally, according to the CCK8 assays, CQ treatment significantly increased the sensitivity of SGC7901/DDP cells to chemotherapeutic drugs (Fig. 4E). In summary, ATG16L1 increased autophagic activity, weakening the sensitivity of chemoresistant GC cells to chemotherapy. 

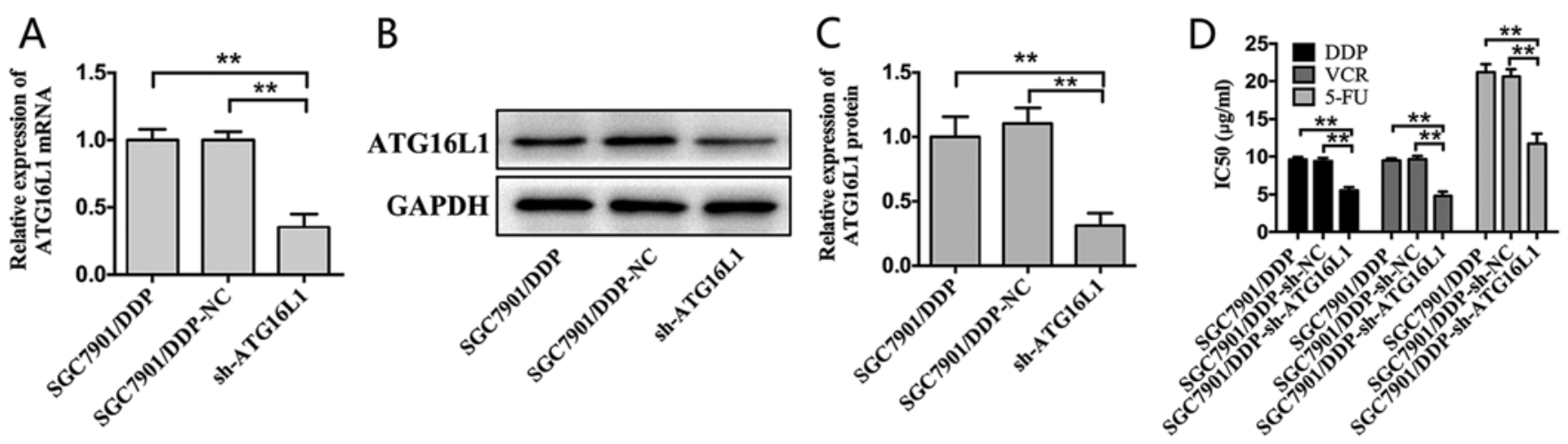

Figure 3. Reduced expression of ATG16L1 increases the sensitivity of SGC7901/DDP cells to chemotherapeutic agents. (A) SGC7901/DDP cells were transfected with sh-ATG16L1 or NC, and the expression of ATG16L1 was then detected by RT-qPCR. (B) The expression of ATG16L1 protein was detected when sh-ATG16L1 or NC were transfected into SGC7901/DDP cells. (C) The histogram shows the relative protein expression of ATG16L1. (D) The IC ${ }_{50}$ values were detected after sh-ATG16L1 or NC were transfected into SGC7901/DDP cells. ${ }^{* *} \mathrm{P}<0.01$. DDP, cisplatin; NC, negative control; sh, short hairpin RNA; ATG16L 1, autophagy-related 16-like 1; VCR, vincristine; 5-FU, 5-fluorouracil.
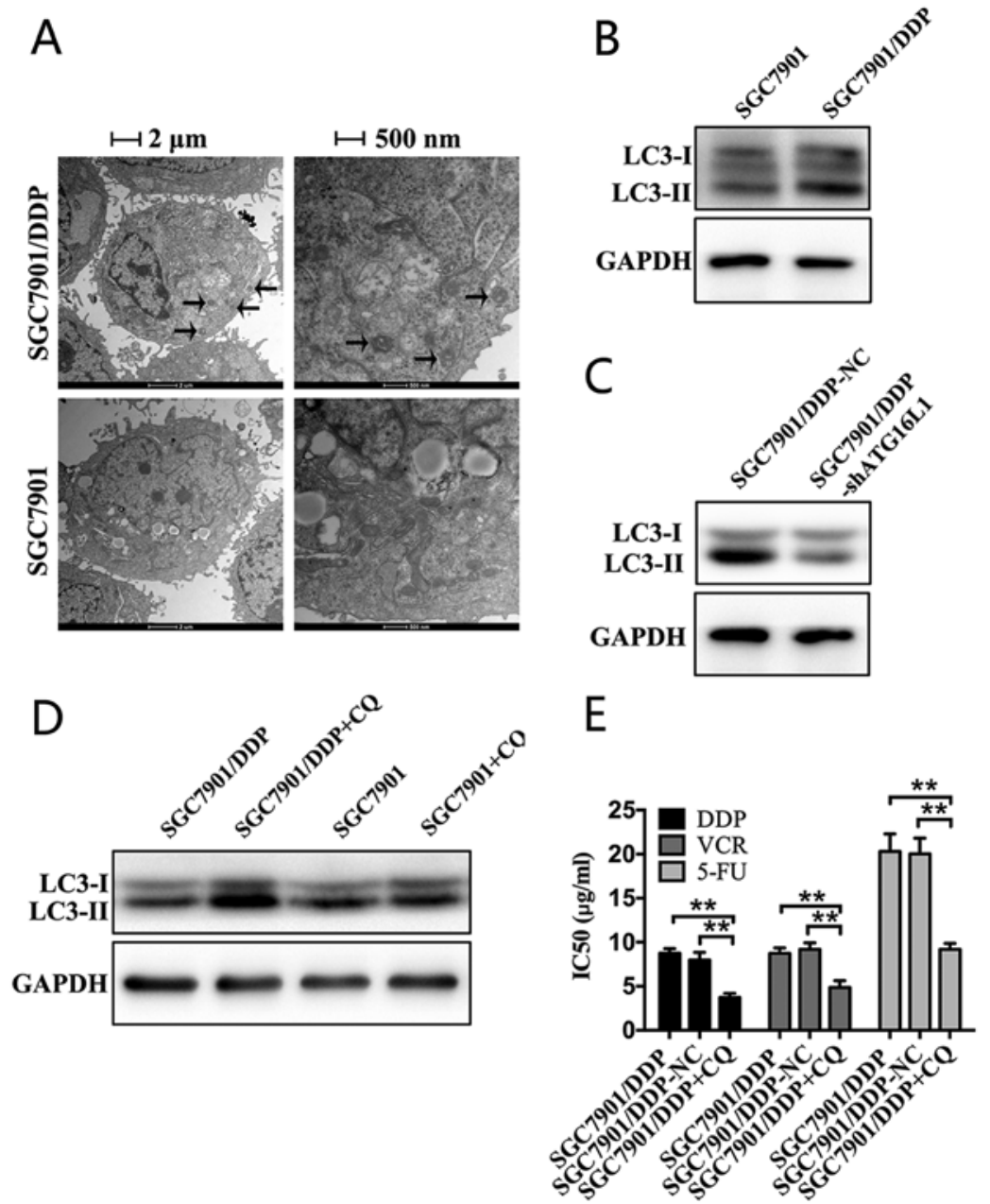

Figure 4. Chemoresistant GC cells (SGC7901/DDP) exhibit increased autophagy, and the inhibition of autophagy restores the sensitivity of chemoresistant cells to chemotherapy. (A) Transmission electron microscopy was performed to evaluate autophagy in SGC7901 and SGC7901/DDP cells. (B) The expression level of LC3 was detected by western blotting in SGC7901 and SGC7901/DDP cells. (C) A western blot assay was performed to evaluate the effect of shRNA targeting ATG16L1 on LC3 expression in SGC7901/DDP cells. (D) SGC7901 and SGC7901/DDP cells treated with or without CQ (20 $\mu \mathrm{mol} / \mathrm{l})$ for $24 \mathrm{~h}$. Subsequently, the expression of LC3 was evaluated by western blotting. (E) CQ enhanced the drug sensitivity of SGC7901/DDP cells to chemotherapy. Arrow: partial autophagosome. ${ }^{* *} \mathrm{P}<0.01$. DDP, cisplatin; CQ, chloroquine; VCR, vincristine; 5-FU, 5-fluorouracil; sh, short hairpin RNA; NC, negative control; ATG16L1, Autophagy-related 16-like 1.

miR-874 inhibits autophagy by regulating ATG16L1. The aforementioned results suggested that ATG16L1 may be a target gene of miR-874 and that ATG16L1 regulated autophagy. Next, we hypothesized that the suppression of miR-874 


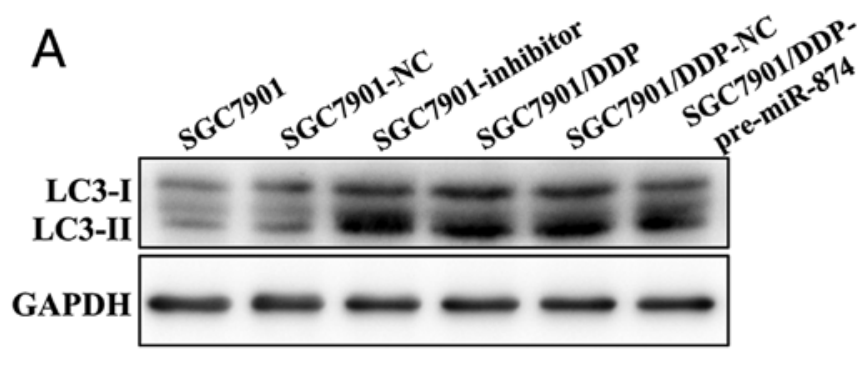

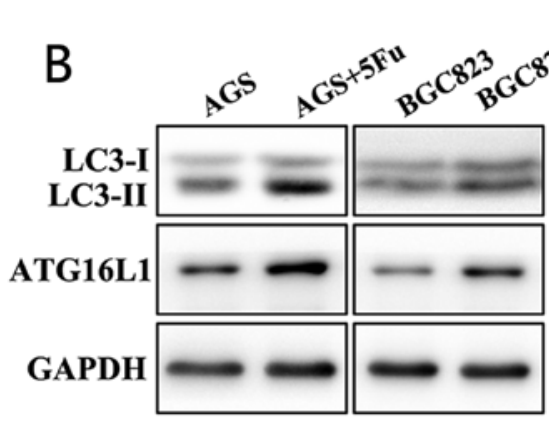

E

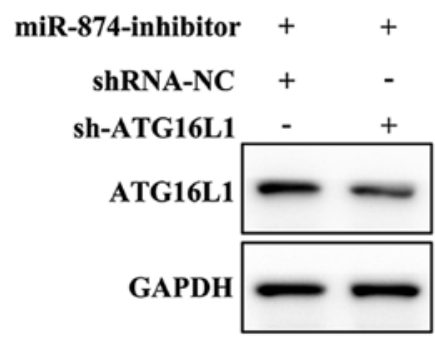

$\mathrm{F}$
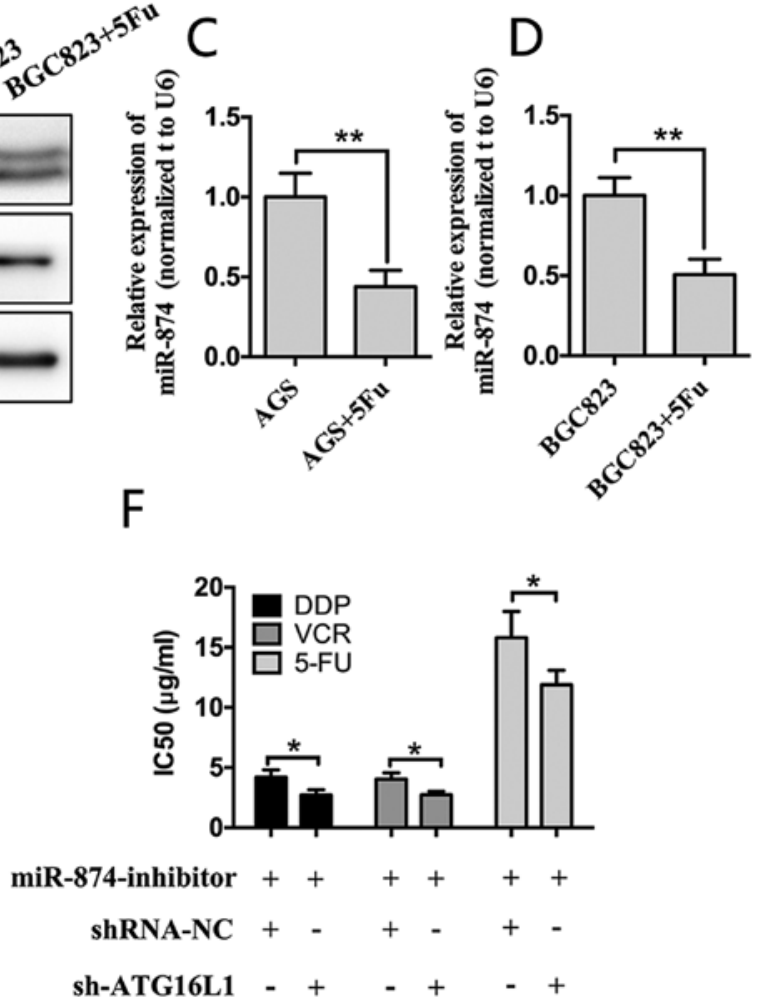

Figure 5. miR-874 inhibits autophagy in chemoresistant cells by negatively regulating ATG16L1. (A) Expression of LC3 was evaluated by western blotting after miR-874 was overexpressed in SGC7901/DDP cells or inhibited in SGC7901 cells. (B) Western blot assay was conducted to detected the protein expression of LC3I/II and ATG16L1 in AGS and BGC823 cells, and after treatment with a low concentration of 5-Fu. (C) The expression of miR-874 was detected in AGS and AGS+5-Fu Cells. (D) The expression of miR-874 was detected in BGC823 and BGC823+5-Fu Cells. (E) SGC7901 cells were co-transfected with miR-874 inhibitors and shRNA-ATG16L1. ATG16L1 was then evaluated by western blot analysis. (F) The IC $_{50}$ values were detected when sh-ATG16L1 or NC was co-transfected with miR-874-inhibitors. ${ }^{*} \mathrm{P}<0.05$ and ${ }^{* *} \mathrm{P}<0.01$. DDP, cisplatin; VCR, vincristine; 5-FU, 5-fluorouracil; sh, short hairpin RNA; NC, negative control; ATG16L1, Autophagy-related 16-like 1; miR, microRNA.

contributes to increased autophagy. To examine this hypothesis, SGC7901/DDP or SGC7901 cells were transfected with pre-miR-874 or inhibitors. Following transfection, autophagy was detected in the aforementioned cells. Downregulation of miR-874 markedly increased the expression of LC3-II in SGC7901 cells compared with the blank and NC groups. In contrast, overexpression of miR-874 led to the opposite effect in SGC7901/DDP cells (Fig. 5A). Furthermore, according to the RT-qPCR and western blot analyses, following treatment of the cells with a low concentration of 5-FU for $24 \mathrm{~h}$, the expression of miR-874 in AGS and BGC823 cells was significantly reduced, whereas the expression levels of ATG16L1 and LC3-II were markedly upregulated (Fig. 5B-D). SGC7901 cells were then transfected with miR-874 inhibitors and shRNAs targeting ATG16L1. Downregulation of ATG16L1 attenuated the effects of the miR-874 inhibitors compared with NC group (Fig. 5E and F). These results confirmed that miR-874 inhibited autophagy by targeting ATG16L1.
Restoration of miR-874 increases chemosensitivity in GC cells in vivo. To determine whether miR-874 affected the chemosensitivity ofGCcellsinvivo, weconstructedSGC7901/DDP cells that stably overexpressed miR-874. SGC7901/DDP-NC or SGC7901/DDP-pre-miR-874 cells were then transplanted into nude mice. The volumes of the pre-miR-874-transfected tumours were significantly decreased following chemotherapy compared with the SGC7901/DDP-NC group, indicating that ectopic miR-874 expression reverted chemoresistance (Fig. 6).

miR-874 expression is downregulated in chemoresistant patients and is associated with overall survival in patients with $G C$. RT-qPCR and immunohistochemistry were performed to detect miR-874 and its targets, respectively, in clinical samples. A total of 50 clinical samples (25 chemoresistant samples and 25 chemosensitive samples) were obtained from patients who received neoadjuvant chemotherapy prior to surgery between 2014 and 2016 at the Affiliated Huai'an Hospital, Xuzhou 
A
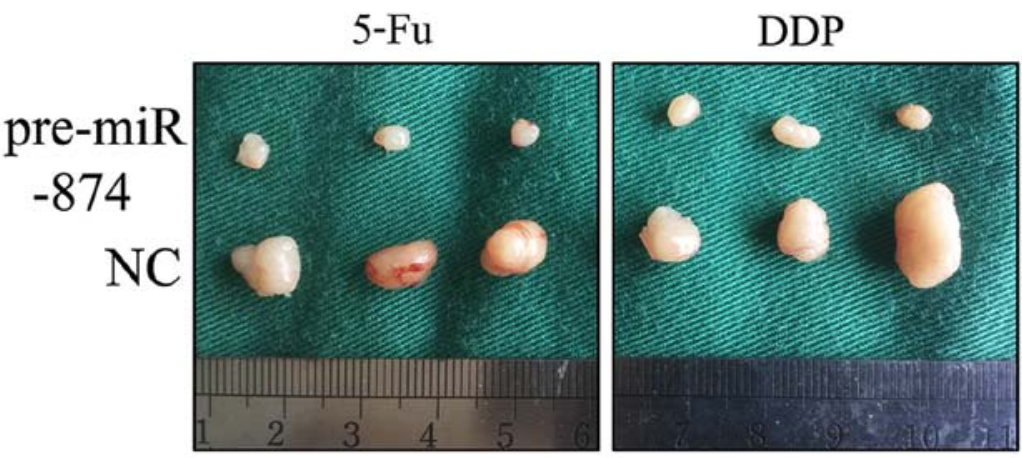

$\mathrm{B}$
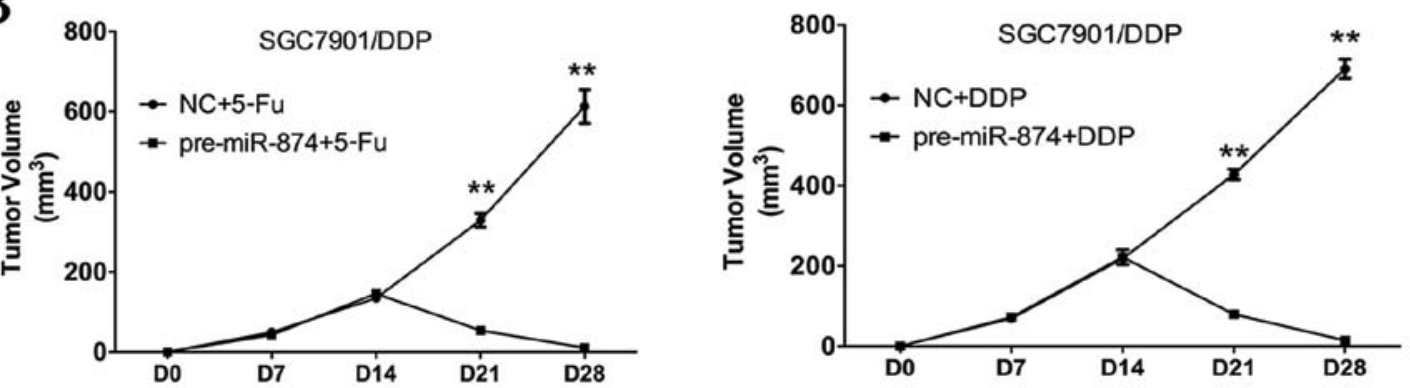

Figure 6. Restoration of miR-874 reverses chemoresistance in vivo. (A) SGC7901/DDP-NC or SGC7901/DDP-pre-miR-874 cells were transplanted into nude mice. (B) Tumour volumes were measured and calculated as length $\mathrm{x}$ width ${ }^{2}$ at the indicated time points. ${ }^{* *} \mathrm{P}<0.01$. DDP, cisplatin; sh, short hairpin RNA; $\mathrm{NC}$, negative control; miR, microRNA; 5-FU, 5-fluorouracil.

A

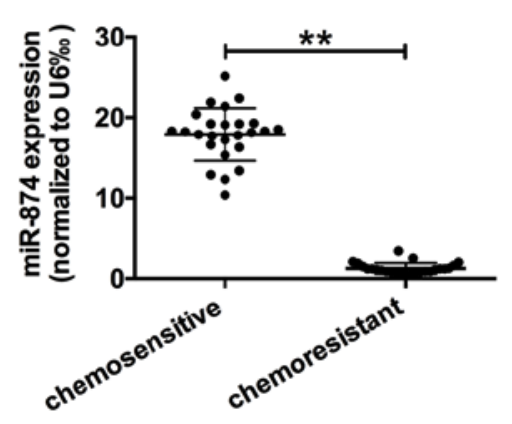

B

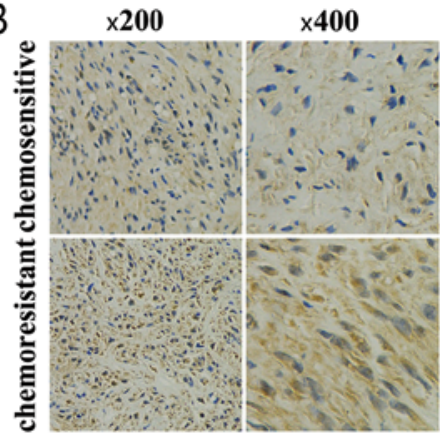

C

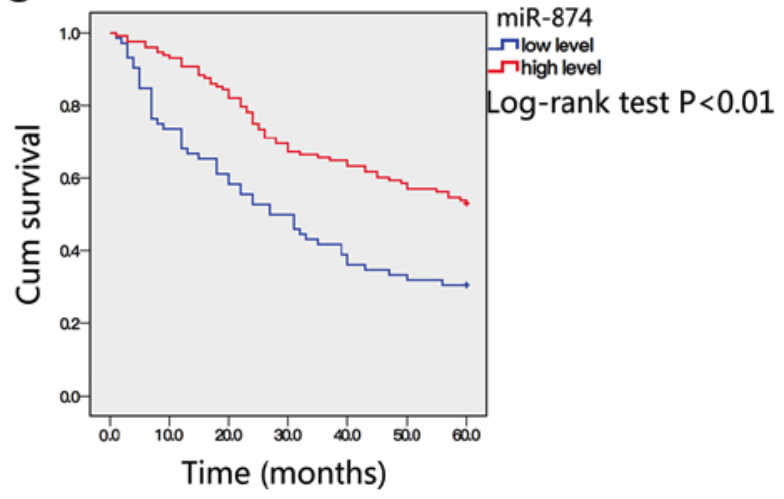

Figure 7. miR-874 expression is inversely associated with the expression of its target gene and is associated with overall survival in patients with gastric cancer. (A) RT-qPCR was conducted to detected the expression of miR-874 in chemosensitive and chemoresistant tissue samples. (B) Immunohistochemistry assay was used to evaluated the ATG16L1 expression in chemosensitive and chemoresistant tissue samples. (C) Kaplan-Meier curves were generated to analyse the association between miR-874 expression and overall survival. ${ }^{* *} \mathrm{P}<0.01$. miR, microRNA.

Medical University. Chemosensitivity or resistance was the result of clinical multidisciplinary discussions. In drug-resistant patients, the expression of miR-874 was significantly decreased and the expression of ATG16L1 was markedly upregulated compared with drug-sensitive patients (Fig. 7A and B). The association between miR-874 expression and clinicopathological parameters from 200 cases of gastric malignant tissues were analysed from Tissue Sample Center, which demonstrated that miR-874 expression was significantly associated with distant metastasis, whereas no significant association was observed between miR-874 expression and patient age, sex, tumour differentiation, tumour depth, nodal metastasis or tumour node metastasis stage (Table I). In addition, patients with low miR-874 expression had a significantly poorer prognosis compared with those with high expression (Fig. 7C).

\section{Discussion}

GC is among the most common causes of cancer-associated mortality. Currently, the rates of early detection and diagnosis among patients with GC are low, and chemotherapy remains a primary treatment method. However, due to drug resistance, the effectiveness of chemotherapy is limited. The 5-year survival rate of patients with $\mathrm{GC}$ remains low $(3,23)$. Although numerous mechanisms contribute to chemoresistance, including increased drug efflux, inactivation of detoxification enzymes, alterations 
Table I. Association between miR-874 expression and clinicopathological characteristics.

\begin{tabular}{|c|c|c|c|c|}
\hline \multirow[b]{2}{*}{ Variables } & \multirow[b]{2}{*}{ No. of cases } & \multicolumn{2}{|c|}{ Expression of miR-874 } & \multirow[b]{2}{*}{ P-value } \\
\hline & & Low $(\%)$ & High $(\%)$ & \\
\hline Age, years & & & & 0.64 \\
\hline$\geq 50$ & 151 & $53(35.1)$ & $98(64.9)$ & \\
\hline$<50$ & 49 & $20(40.8)$ & $29(59.2)$ & \\
\hline Sex & & & & 0.22 \\
\hline Male & 111 & $31(27.9)$ & $80(72.1)$ & \\
\hline Female & 89 & $32(36.0)$ & $57(64.0)$ & \\
\hline Degree of differentiation & & & & 0.38 \\
\hline Well and moderately differentiated & 171 & $62(36.3)$ & $109(63.7)$ & \\
\hline Poorly differentiated & 29 & $13(44.8)$ & $16(55.2)$ & \\
\hline $\mathrm{T}$ classification & & & & 0.13 \\
\hline $\mathrm{T} 1$ & 14 & $6(42.9)$ & $8(57.1)$ & \\
\hline $\mathrm{T} 2$ & 58 & $15(25.9)$ & $43(74.1)$ & \\
\hline $\mathrm{T} 3$ & 116 & $43(37.1)$ & $73(62.9)$ & \\
\hline $\mathrm{T} 4$ & 12 & $7(58.3)$ & $5(41.7)$ & \\
\hline $\mathrm{N}$ classification & & & & 0.68 \\
\hline N0 & 119 & $49(41.2)$ & $70(58.8)$ & \\
\hline $\mathrm{N} \geq 1$ & 81 & $31(38.3)$ & $50(61.7)$ & \\
\hline M classification & & & & $<0.01$ \\
\hline M0 & 166 & $52(31.3)$ & $114(68.7)$ & \\
\hline M1 & 34 & $21(61.8)$ & $13(38.2)$ & \\
\hline TNM stage & & & & 0.12 \\
\hline Stage I/II & 121 & $39(32.2)$ & $82(67.8)$ & \\
\hline Stage III/IV & 79 & $34(43.0)$ & $45(57.0)$ & \\
\hline
\end{tabular}

TNM, tumour node metastasis; miR, microRNA.

in drug metabolism, mutations of drug targets, dysfunction of pro-apoptotic proteins and enhancement of DNA repair activity, the mechanisms involved in cancer chemoresistance remain poorly understood (24-28). Evidence suggests that miRNAs are involved in chemoresistance in various types of cancer, including GC $(6,14)$. However, relatively few studies investigating MDR in GC have been performed.

The dysregulation of miR-874 has been reported in various cancer types, as aforementioned. In the present study, the underlying mechanisms through which miR-874 regulates MDR in GC were studied. miR-874 expression was demonstrated to be decreased in MDR GC cells compared with parental GC cells, indicating that miR-874 is involved in chemoresistance.

miRNAs usually have multiple target genes. Therefore, a search for potential target genes of miR-874 in GC using several computational algorithms was performed. Among the potential targets, ATG16L1 was focused on as its function is associated with chemoresistance in cancer (29). The results of the present study revealed that the expression of ATG16L1 was increased in drug-resistant GC cells. Furthermore, miR-874 reduced ATG16L1 expression at the mRNA and protein levels. Knockdown of ATG16L1 expression by shRNA increased the chemosensitivity of chemoresistant GC cells, suggesting that ATG16L1 is associated with chemosensitivity in GC.

Autophagy is increased in various cancer types and contributes to drug resistance. ATG16L1 is a member of a large protein complex that is necessary for autophagy, which is the major process by which intracellular components are targeted to lysosomes for degradation $(30,31)$. The expression of ATG16L1 was increased in MDR GC cells, suggesting that autophagy may be involved in MDR. To examine this hypothesis, the level of autophagy was evaluated by performing TEM and western blot analyses. The results demonstrated that MDR cells exhibited increased autophagy, which functioned as a mechanism of chemoresistance. The level of autophagy decreased following the knockdown of ATG16L1 or treating MDR cells with CQ, both of which resulted in increased sensitivity to chemotherapy agents. These results suggest that autophagy in MDR GC cells may be a mechanism that promotes chemotherapy resistance. The inhibition of autophagy by interfering with ATG16L1 may be a novel approach to improving chemotherapeutic efficacy.

miRNAs serve an important role in regulating autophagy $(32,33)$. The expression of miR-874 was modified 
by transfecting GC cells with pre-miR-874 or inhibitors to ascertain whether miR-874 regulated autophagy. The upregulation of miR-874 significantly inhibited autophagy in MDR cells, while the downregulation of miR-874 had the opposite effect. To verify whether these effects were mediated by ATG16L1, SGC7901 cells were co-transfected with miR-874 inhibitors and shRNAs targeting ATG16L1. The downregulation of ATG16L1 by shRNAs reversed the effect of miR-874 inhibition on autophagy. Taken together, these findings indicated that miR-874 inhibits autophagy by targeting ATG16L1 in MDR cells, highlighting the potential of miR-874 as a target in human GC therapy.

To determine the effects of miR-874 in the regulation of drug resistance in vivo, a xenograft tumour model and clinical GC specimens were used. The results confirmed that miR-874 also regulates drug resistance in vivo. In addition, miR-874 was not associated with any clinicopathological parameters except distant metastasis. Furthermore, according to a Kaplan Meier analysis, the prognosis of patients with GC was associated with the expression level of miR-874. Thus, miR-874 may serve as an MDR marker in GC to guide chemotherapy and may be used as a predictor of overall survival.

miR-874 was also demonstrated to be significantly downregulated in chemoresistant cells and GC tissue samples. To further explore whether chemotherapeutic agents influenced the expression of miR-874, two typical gastric adenocarcinoma cell lines, AGS and BGC823, were treated with 5-FU, which is widely used in clinical settings as a chemotherapeutic agent. The results revealed that 5-FU treatment significantly decreased the expression of miR-874. Further studies are required to determine the exact effects of chemotherapy on the expression of miR-874.

In summary, the results of the current study suggest that miR-874 is a novel miRNA, which regulates MDR in GC. miR-874 inhibits autophagy by targeting ATG16L1 in MDR cells, leading to increased chemotherapeutic sensitivity. These findings reveal a novel miR-874/ ATG16L1/autophagy/chemosensitivity regulatory axis. Furthermore, this study has clinical relevance as miR-874 overexpression and/or strategies that inhibit autophagy may have potential therapeutic applications for the treatment of MDR in GC.

\section{Acknowledgements}

Not applicable.

\section{Funding}

This study was funded by a Huai'an International Science and Technology Cooperation Research Project (grant no. HAC201709), the 333 High-Level Talents Training Project of Jiangsu Province (grant no. BRA2017247), and a Jiangsu Province Young Medical Talent Project (grant no. QNRC 2016423).

\section{Availability of data and materials}

The datasets used and/or analysed in the current study are available from the corresponding author on reasonable request.

\section{Authors' contributions}

$\mathrm{HH}$, JT and LZ designed and performed the experiments and contributed to the data analysis; YB enrolled the patients, measured the RNA levels in the clinical samples and analysed the relevant data; XZ initiated the work and wrote the manuscript; and all authors read and approved the final version of the manuscript.

\section{Ethics approval and consent to participate}

The use of human tissues was approved by the Ethics Committee of the Affiliated Huai'an Hospital of Xu Zhou Medical University, and patient consent was obtained.

\section{Patient consent for publication}

Not applicable.

\section{Competing interests}

The authors declare that they have no competing interests.

\section{References}

1. Torre LA, Bray F, Siegel RL, Ferlay J, Lortet-Tieulent J and Jemal A: Global cancer statistics, 2012. CA Cancer J Clin 65: 87-108, 2015.

2. Van Cutsem E, Sagaert X, Topal B, Haustermans K and Prenen H: Gastric cancer. Lancet 388: 2654-2664, 2016.

3. Charalampakis N, Economopoulou P, Kotsantis I, Tolia M, Schizas D, Liakakos T, Elimova E, Ajani JA and Psyrri A: Medical management of gastric cancer: A 2017 update. Cancer Med 7: 123-133, 2018.

4. Sitarz R, Skierucha M, Mielko J, Offerhaus GJA, Maciejewski R and Polkowski WP: Gastric cancer: Epidemiology, prevention, classification, and treatment. Cancer Manag Res 10: 239-248, 2018.

5. Araújo R, Santos JM, Fernandes M, Dias F, Sousa H, Ribeiro J, Bastos MM, Oliveira PA, Carmo D, Casaca F, et al: Expression profile of microRNA-146a along HPV-induced multistep carcinogenesis: A study in HPV16 transgenic mice. J Cancer Res Clin Oncol 144: 241-248, 2018.

6. Dehghanzadeh R, Jadidi-Niaragh F, Gharibi T and Yousefi M: MicroRNA-induced drug resistance in gastric cancer. Biomed Pharmacother 74: 191-199, 2015.

7. Mohammadi A, Mansoori B and Baradaran B: The role of microRNAs in colorectal cancer. Biomed Pharmacother 84: 705-713, 2016.

8. Yang R, Li P, Zhang G, Lu C, Wang H and Zhao G: Long non-coding RNA XLOC_008466 functions as an oncogene in human non-small cell lung cancer by targeting miR-874. Cell Physiol Biochem 42: 126-136, 2017.

9. Shea A, Harish V, Afzal Z, Chijioke J, Kedir H, Dusmatova S, Roy A, Ramalinga M, Harris B, Blancato J, et al: MicroRNAs in glioblastoma multiforme pathogenesis and therapeutics. Cancer Med 5: 1917-1946, 2016

10. Jin F, Wang Y, Li M, Zhu Y, Liang H, Wang C, Wang F, Zhang CY, Zen K and Li L: MiR-26 enhances chemosensitivity and promotes apoptosis of hepatocellular carcinoma cells through inhibiting autophagy. Cell Death Dis 8: e2540, 2017.

11. Zhang B, Ji S, Ma F, Ma Q, Lu X and Chen X: miR-489 acts as a tumor suppressor in human gastric cancer by targeting PROX1. Am J Cancer Res 6: 2021-2030, 2016.

12. Wei T, Zhu W, Fang S, Zeng X, Huang J, Yang J, Zhang J and Guo L: miR-495 promotes the chemoresistance of SCLC through the epithelial-mesenchymal transition via Etk/BMX. Am J Cancer Res 7: 628-646, 2017.

13. Riquelme I, Letelier P, Riffo-Campos AL, Brebi P and Roa JC: Emerging Role of miRNAs in the Drug Resistance of Gastric Cancer. Int J Mol Sci 17: 424, 2016. 
14. Calıskan M, Güler H and Bozok Cetintas V: Current updates on microRNAs as regulators of chemoresistance. Biomed Pharmacother 95: 1000-1012, 2017.

15. Nohata N, Hanazawa T, Kinoshita T, Inamine A, Kikkawa N, Itesako T, Yoshino H, Enokida H, Nakagawa M, Okamoto Y, et al: Tumour-suppressive microRNA-874 contributes to cell proliferation through targeting of histone deacetylase 1 in head and neck squamous cell carcinoma. Br J Cancer 108: 1648-1658, 2013.

16. Que K, Tong Y, Que G, Li L, Lin H, Huang S, Wang R and Tang L: Downregulation of miR-874-3p promotes chemotherapeutic resistance in colorectal cancer via inactivation of the Hippo signaling pathway. Oncol Rep 38: 3376-3386, 2017.

17. Wang L, Gao W, Hu F, Xu Z and Wang F: MicroRNA-874 inhibits cell proliferation and induces apoptosis in human breast cancer by targeting CDK9. FEBS Lett 588: 4527-4535, 2014.

18. Zhang X, Tang J, Zhi X, Xie K, Wang W, Li Z, Zhu Y, Yang L, $\mathrm{Xu} \mathrm{H}$ and $\mathrm{Xu} \mathrm{Z}$ : miR-874 functions as a tumor suppressor by inhibiting angiogenesis through STAT3/VEGF-A pathway in gastric cancer. Oncotarget 6: 1605-1617, 2015.

19. Brierley JD, Gospodarwicz MK and Wittekind C (eds): TNM classification of maligant tumours. 8th edition. Wiley Blackwell, Oxford, 2017.

20. Amin MB, Edge SB, Greene FL and Brierley JD: AJCC cancer staging manual. 8th edition. Springer, New York, 2017.

21. Livak KJ and Schmittgen TD: Analysis of relative gene expression data using real-time quantitative PCR and the 2(-Delta Delta C(T)) method. Methods 25: 402-408, 2001.

22. Lu Y, Gao J, Zhang S, Gu J, Lu H, Xia Y, Zhu Q, Qian X, Zhang F, Zhang C, et al: miR-142-3p regulates autophagy by targeting ATG16L1 in thymic-derived regulatory T cell (tTreg). Cell Death Dis 9: 290,2018

23. Lee KW, Lee JH, Kim JW, Kim JW, Ahn S and Kim JH: Population-based outcomes research on treatment patterns and impact of chemotherapy in older patients with metastatic gastric cancer. J Cancer Res Clin Oncol 142: 687-697, 2016.

24. Annovazzi L, Mellai M and Schiffer D: Chemotherapeutic drugs: DNA damage and repair in glioblastoma. Cancers (Basel) 9: 57 , 2017.
25. Adamska A, Elaskalani O, Emmanouilidi A, Kim M, Abdol Razak NB, Metharom P and Falasca M: Molecular and cellular mechanisms of chemoresistance in pancreatic cancer. Adv Biol Regul 68: 77-87, 2017.

26. Bourguignon LY, Earle $C$ and Shiina M: Activation of matrix hyaluronan-mediated CD44 signaling, epigenetic regulation and chemoresistance in head and neck cancer stem cells. Int J Mol Sci 18: 18, 2017.

27. Butera G, Pacchiana R and Donadelli M: Autocrine mechanisms of cancer chemoresistance. Semin Cell Dev Biol 78: 3-12, 2017.

28. de Oliveira Júnior RG, Christiane Adrielly AF, da Silva Almeida JR, Grougnet R, Thiéry V and Picot L: Sensitization of tumor cells to chemotherapy by natural products: A systematic review of preclinical data and molecular mechanisms. Fitoterapia 129: 383-400, 2018.

29. Zhang K, Chen J, Zhou H, Chen Y, Zhi Y, Zhang B, Chen L, Chu X, Wang R and Zhang C: PU.1/microRNA-142-3p targets ATG5/ATG16L1 to inactivate autophagy and sensitize hepatocellular carcinoma cells to sorafenib. Cell Death Dis 9: 312, 2018.

30. Bhat P, Kriel J, Shubha Priya B, Basappa, Shivananju NS and Loos B: Modulating autophagy in cancer therapy: Advancements and challenges for cancer cell death sensitization. Biochem Pharmacol 147: 170-182, 2018.

31. Sannigrahi MK, Singh V, Sharma R, Panda NK and Khullar M: Role of autophagy in head and neck cancer and therapeutic resistance. Oral Dis 21: 283-291, 2015.

32. Wu J, Gao F, Xu T, Deng X, Wang C, Yang X, Hu Z, Long Y, He X, Liang G, et al: miR-503 suppresses the proliferation and metastasis of esophageal squamous cell carcinoma by triggering autophagy via PKA/mTOR signaling. Int J Oncol: Mar 16, 2018 (Epub ahead of print). doi: 10.3892/ijo.2018.4320.

33. Wang S, Kobeissi A, Dong Y, Kaplan N, Yang W, He C, Zeng K and Peng H: MicroRNAs-103/107 regulate autophagy in the epidermis. J Invest Dermatol 138: 1481-1490, 2018. 